\title{
Improved results in supersymmetric electroweak baryogenesis
}

\author{
M. Carena ${ }^{\mathrm{a}}$, M. Quirós ${ }^{\mathrm{b}}$, M. Seco ${ }^{\mathrm{c}}$, C.E.M. Wagner ${ }^{\mathrm{d}, \mathrm{e}}$ \\ ${ }^{a}$ Fermi National Accelerator Laboratory, PO Box 500, Batavia, IL 60510, USA \\ b Instituto de Estructura de la Materia (CSIC), Serrano 123, E-28006 Madrid, Spain \\ ${ }^{c}$ Department of Physics, University of Virginia, 382 McCormick Road, Charlottesville, VA 22904-4714, USA \\ d HEP Division, Argonne National Laboratory, 9700 Cass Ave., Argonne, IL 60439, USA \\ e Enrico Fermi Institute, University of Chicago, 5640 Ellis Ave., Chicago, IL 60637, USA
}

Received 12 August 2002; received in revised form 24 October 2002; accepted 22 November 2002

\begin{abstract}
Electroweak baryogenesis provides a very attractive scenario to explain the origin of the baryon asymmetry. The mechanism of electroweak baryogenesis makes use of the baryon number anomaly and relies on physics that can be tested experimentally. It is today understood that, if the Higgs mass is not larger than $120 \mathrm{GeV}$, this mechanism may be effective within supersymmetric extensions of the Standard Model. In this work, we reconsider the question of baryon number generation at the electroweak phase transition within the context of the minimal supersymmetric extension of the Standard Model. We derive the relevant diffusion equations, give a consistent definition of the sources, and compare our results with those appearing in the recent literature on this subject.

(C) 2002 Elsevier Science B.V. All rights reserved.
\end{abstract}

\section{Introduction}

Electroweak baryogenesis [1] provides a predictive framework for the computation of the baryon asymmetry of the Universe [2]. Perhaps the most attractive feature of this mechanism is that it relies on anomalous baryon number violation processes which are present in the Standard Model [3]. At temperatures far above the electroweak phase transition scale, these anomalous processes are unsuppressed and, in the absence of any

\footnotetext{
Work supported in part by CICYT, Spain, under contract FPA2001-1806, and by EU under contracts HPRNCT-2000-00152 and HPRN-CT-2000-00148.

E-mail address: mariano@makoki.iem.csic.es (M. Quirós).
} 
$B-L$ asymmetry, they lead to the erasure of any baryon or lepton number generated at high energy scales [4]. These baryon number violation processes are, instead, exponentially suppressed in the electroweak symmetry broken phase, at temperatures far below the electroweak phase transition [5]. At the electroweak phase transition, non-equilibrium processes may generate a non-vanishing baryon number which may efficiently diffuse into the broken phase [6]. The generated baryon number depends on the CP-violating currents present in the model. The mechanism of electroweak baryogenesis may become effective if the $\mathrm{CP}$-violating sources are strong and, at the electroweak phase transition temperature, the baryon number violation processes in the broken phase are sufficiently suppressed, leading to a baryon number density in the broken phase consistent with observations. This, in turn, demands a strongly first order electroweak phase transition.

It has been long ago realized that in the Standard Model the CP violating sources are too weak to lead to an acceptable baryon number density [7,8]. Moreover, even if the sources were strong enough to lead to a reasonable baryon number generation, for the Higgs masses consistent with the present experimental constraints the electroweak phase transition is a cross-over. Hence, the baryon number violating processes are in equilibrium at the electroweak phase transition and leading to no baryon number generation in the broken phase [9]. A minimal supersymmetric extension of the Standard Model (MSSM), instead, has all the necessary ingredients to improve on both problems [10]. First, there are additional sources of CP-violation, associated with the CP-violating phases of the supersymmetry breaking parameters. Second, in the presence of a light stop, the phase transition may become strong enough to allow the preservation of the baryon number generated at the electroweak symmetry breaking scale [11-28].

Although there is general agreement on both the existence and nature of the new sources and also on the parameter space leading to a strongly first order phase transition, the results regarding the strength and specific form of the $\mathrm{CP}$-violating sources are still controversial. Most groups working on the subject have found that, for values of the CP-odd Higgs mass of the order of the weak scale, the sources are dominated by a term proportional to $\epsilon_{i j} H_{i} \partial^{\mu} H_{j}$, where $H_{i}$ denote the expectation value of the neutral components of the two Higgs doublets present at low energies [29-39]. Recently, however, it has been claimed that this contribution is absent, leading to a suppressed result for the baryon asymmetry contribution within the MSSM proportional to $H_{1} \partial^{\mu} H_{2}+(1 \leftrightarrow 2)$ [40-42].

In this article, we proceed to perform a critical reanalysis of the diffusion equations leading to the baryon asymmetry and of the sources for those diffusion equations. In particular, we demonstrate that the sources can be expressed in terms of appropriate differential operators times the CP-violating currents we computed in a very recent work. We find no suppression of the dominant sources proportional to $\epsilon_{i j} H_{i} \partial^{\mu} H_{j}$. Indeed, we show that the suppression claimed by the authors of Refs. [40-42] is an artifact of the approximation they used in order to compute the currents.

The organization of the article is as follows. In Section 2 we provide the derivation of the diffusion equations. In Section 3 we review the derivation of the CP-violating currents and we show explicitly where our results differ from the ones of Ref. [41]. In Section 4 we discuss the results for the baryon asymmetry within the MSSM and Section 5 contains our conclusions. Finally some useful formulae concerning the chargino sector of the MSSM are summarized in Appendix A. 


\section{Diffusion equations}

We will start considering a system of particles propagating in a non-trivial CP-violating background localized in the bubble wall, where the bubble is expanding with a speed $v_{\omega}$ in the plasma frame. In the absence of a complete treatment of quantum Boltzmann equations we will use the quasi-classical approximation and will keep all quantum corrections in the calculation of the sources, which has been done using thermal field theory (see Section 3). Classical Boltzmann equations are expected to give a sufficiently good approximation and will allow us to compare with the results of Ref. [41] where this approach has also been adopted.

The presence of the CP-violating background and the particle number changing reactions perturb the distribution functions for particles and antiparticles around the equilibrium. The corresponding distribution functions $f_{i}$ satisfy the Boltzmann equations

$$
v^{\mu} \partial_{\mu} f_{i}+\bar{F}_{i}^{\mu} \nabla_{\mu} f_{i}=C_{i}[f],
$$

where we are using the notation $\partial_{\mu} \equiv \partial / \partial x^{\mu}, \nabla_{\mu} \equiv \partial / \partial p^{\mu}, v^{\mu}=d x^{\mu} / d t \equiv p^{\mu} / E$ is the four-velocity and $\bar{F}^{\mu}=d p^{\mu} / d t$ is the force generated by the non-trivial background. The term on the right-hand side $C_{i}=\left(\partial f_{i} / \partial t\right)_{\text {coll }}$ encodes the effect on the distribution of the particle number changing reactions and elastic collisions.

In the bubble-wall frame and neglecting the curvature of the bubble wall the distribution functions can be written as ${ }^{1}$

$$
f_{i}^{P}=\frac{\bar{g}\left(p_{z}, z\right)}{e^{\beta\left[E+v_{\omega} p_{z}-\mu_{i}^{P}\right]} \pm 1}+\delta f_{i}^{P},
$$

where $\mu_{i}^{P}=\mu_{i}^{P}(z)$ is the chemical potential, $E$ and $p_{z}$ are the energy and momentum of the particle, the momentum-dependent part $\delta f_{i}^{P}=\delta f_{i}^{P}\left(E, p_{z} ; z\right)$ describes departure of the system from kinetic equilibrium and we have introduced a slowly varying CP-even function of $p_{z}$ and $z, \bar{g}\left(p_{z}, z\right)$, which tends to 1 in the absence of forces. Considering the effective distribution functions $f_{i}=f_{i}^{P}-f_{i}^{\bar{P}}$, describing the difference between particles and antiparticles of a given species, and applying (2.2) to Eq. (2.1) gives

$$
\frac{p_{z}}{E}\left[-\mu_{i}^{\prime} \bar{g}\left(p_{z}, z\right) \frac{\partial f_{0}}{\partial E}+\delta f_{i}^{\prime}\right]+\left(F_{i}^{0}+v_{\omega} F_{i}^{z}\right) \bar{g}\left(p_{z}, z\right) \frac{\partial f_{0}}{\partial E}=C_{i},
$$

where $F_{i}^{\mu}$ is the CP-violating component of the force, we have linearized with respect to the small perturbations, $\mu_{i}, \delta f_{i}$, in the spirit of the Chapman-Enskog first order approximation [45], $f_{0}$ is the equilibrium distribution function in the plasma frame, and the $\left(^{\prime}\right)$ denotes derivative with respect to $z$. In this process, due to the assumed smoothness of the CP-even function $\bar{g}\left(p_{z}, z\right)$, we have ignored terms proportional to derivatives of this function.

\footnotetext{
1 The ansatz for distribution functions is parametrized in terms of kinetic momenta. For a discussion between the formalisms using kinetic versus canonical momenta see Ref. [41].
} 
Following Ref. [41], we rewrite the above equation in the plasma frame by performing a Galilean transformation on the velocity $v_{z}$ to $v_{z}-v_{\omega}$,

$$
\left(\frac{p_{z}}{E}-v_{\omega}\right)\left[-\mu_{i}^{\prime} g\left(p_{z}, z\right) \frac{\partial f_{0}}{\partial E}+\delta f_{i}^{\prime}\right]+\left(F_{i}^{0}+v_{\omega} F_{i}^{z}\right) g\left(p_{z}, z\right) \frac{\partial f_{0}}{\partial E}=C_{i},
$$

where $g\left(p_{z}, z\right)$ is assumed to be an even function of momenta in this frame.

We now define

$$
\langle X\rangle \equiv \int \frac{d^{3} p}{(2 \pi)^{3}} X
$$

and using the decomposition

$$
f_{i}=-\mu_{i} \frac{\partial f_{0}}{\partial E} g\left(p_{z}, z\right)+\delta f_{i}
$$

and the definition of the current

$$
j_{i}^{\mu} \equiv\left\langle v^{\mu} f_{i}\right\rangle=\left(n_{i}, j_{i}^{z}\right)
$$

we can relate the $\mathrm{CP}$-violating current with the chemical potential and the function $\delta f_{i}$ as,

$$
\begin{aligned}
& n_{i}^{\prime}=-\left\langle\frac{\partial f_{0}}{\partial E} g\left(p_{z}, z\right)\right\rangle \mu_{i}^{\prime}, \\
& j_{i}^{z}=\left\langle\frac{p_{z}}{E} \delta f_{i}\right\rangle,
\end{aligned}
$$

where, again, we have neglected terms proportional to derivatives of $g\left(p_{z}, z\right)$. The function $\delta f_{i}$ is such that $\left\langle\delta f_{i}\right\rangle=0$ and the number density is entirely provided by the chemical potential $\mu_{i}$. In this way we can choose, with all generality, the function $\delta f_{i}$ as odd with respect to $v_{z}$ and therefore satisfying the property $\left\langle v_{z}^{2 n} \delta f_{i}\right\rangle=0$.

We now multiply Eq. (2.4) by $v^{\mu}=\left(1, p_{z} / E\right)$ and integrating over momenta we get the two equations

$$
\begin{aligned}
& -v_{\omega} n_{i}^{\prime}+\left(j_{i}^{z}\right)^{\prime}+\left\langle\left(F_{i}^{0}+v_{\omega} F_{i}^{z}\right) g\left(p_{z}, z\right) \frac{\partial f_{0}}{\partial E}\right\rangle=\left\langle C_{i}\right\rangle, \\
& D_{i} \Gamma_{i}^{T} n_{i}^{\prime}-v_{\omega}\left(j_{i}^{z}\right)^{\prime}+\left\langle\frac{p_{z}}{E}\left(F_{i}^{0}+v_{\omega} F_{i}^{z}\right) g\left(p_{z}, z\right) \frac{\partial f_{0}}{\partial E}\right\rangle=\left\langle\frac{p_{z}}{E} C_{i}\right\rangle,
\end{aligned}
$$

where $\Gamma_{i}^{T}$ is the total interaction rate and the diffusion coefficient $D_{i}$ is defined as

$$
D_{i}=\frac{1}{\Gamma_{i}^{T}} \frac{\left\langle\frac{p_{z}^{2}}{E^{2}} \frac{\partial f_{0}}{\partial E}\right\rangle}{\left\langle\frac{\partial f_{0}}{\partial E}\right\rangle}
$$

and we have again ignored the smooth momentum dependence of $g\left(p_{z}, z\right)$ in the numerator and denominator integral functions. Observe that the ratio (2.11) remains constant up to derivatives of $g^{\prime}\left(p_{z}, z\right)$ which we have consistently ignored in our treatment.

The terms on the right-hand sides of Eqs. (2.9) and (2.10) can be decomposed, using the decomposition (2.6) as

$$
C_{i}[f]=C_{i}^{1}[\mu]+C_{i}^{2}[\delta f],
$$


where $C_{i}^{1}$ and $C_{i}^{2}$ are linear in $\mu_{i}$ and $\delta f_{i}$, respectively. In fact $\left\langle C_{i}^{1}[\mu]\right\rangle$ is entirely provided by inelastic collisions

$$
\left\langle C_{i}^{1}[\mu]\right) \simeq-\Gamma_{i j} \frac{n_{j}}{k_{j}}
$$

where $k_{j}=2\left(k_{j}=1\right)$ for bosons (fermions) are statistical factors, $\Gamma_{i j}\left(j=j_{1}, j_{2}, \ldots\right)$ is the averaged interaction rate for the inelastic reaction channel $(i, j)$ and $n_{j} / k_{j}$ implies a sign over all particles participating in the reaction with appropriate sign assignments: for an inelastic reaction corresponding to the decay $i \rightarrow j_{1} j_{2}$ with decay width $\Gamma_{i j_{1} j_{2}}$, its contribution to the right-hand side of (2.13) would be $-\Gamma_{i j_{1} j_{2}}\left(n_{i} / k_{i}-n_{j_{1}} / k_{j_{1}}-n_{j_{2}} / k_{j_{2}}\right)$. Furthermore $\left\langle C_{i}^{2}[\delta f]\right\rangle=0$ from the oddness of $\delta f_{i}$. On the other hand, $\left\langle v_{z} C_{i}^{1}[\mu]\right\rangle=0$.

Within the present framework, the $\mathrm{CP}$-violating forces are provided by the interaction of the different fields with the Higgs profiles, which vary along the bubble wall. These CPviolating forces induce $\mathrm{CP}$-violating currents for the different fields, which lead to $\left(n_{i}, j_{i}^{z}\right)$ after elastic and inelastic interactions with the other fields present in the plasma. The resulting currents have then two components. The first one, $\left(n_{i}^{(B)}, j_{i}^{(B)}\right)$, the background component, is the one that would be obtained in the presence of only the interaction with the background Higgs field, and the second one, the "collision" component comes from the interaction with the plasma fields and lead to the diffusion process. The integral $\left\langle v_{z} C_{i}^{2}[\delta f]\right\rangle$ is contributed by all collisions and dominated by the elastic ones. Correspondingly with the two components, background and collision, of the currents there can be defined two $\delta f$ components.

The part of $\delta f$ that is induced by the interactions with the plasma fields, the collision component, is governed by the same interactions as the ones governing $\left\langle C_{i}\right\rangle$ and is therefore relevant in the same regime of momenta in which the collision term becomes important. On the other hand the $\delta f$ component coming from the interaction with the background fields, $\delta f^{(B)}$, is not correlated with the collision terms and leads naturally to a negligible contribution to $\left\langle\frac{p_{z}}{E} C_{i}^{2}[\delta f]\right\rangle$. Therefore, considering that the rate of interactions is approximately constant in the momentum regime in which the collision contribution to the currents become relevant, and negligible anywhere else, we get

$$
\left\langle\frac{p_{z}}{E} C_{i}^{2}[\delta f]\right\rangle \simeq \Gamma_{i}^{T}\left(j_{i}^{z}-j_{i}^{(B) z}\right) .
$$

Using now (2.13) and (2.14) in (2.9) and (2.10) we obtain the diffusion equations

$$
\begin{aligned}
& -v_{\omega} n_{i}^{\prime}+\left(j_{i}^{z}\right)^{\prime}+\left\langle\left(F_{i}^{0}+v_{\omega} F_{i}^{z}\right) g\left(p_{z}, z\right) \frac{\partial f_{0}}{\partial E}\right\rangle=-\Gamma_{i, j} \frac{n_{j}}{k_{j}} \\
& D_{i} \Gamma_{i}^{T} n_{i}^{\prime}-v_{\omega}\left(j_{i}^{z}\right)^{\prime}+\left\langle\frac{p_{z}}{E}\left(F_{i}^{0}+v_{\omega} F_{i}^{z}\right) g\left(p_{z}, z\right) \frac{\partial f_{0}}{\partial E}\right\rangle=\Gamma_{i}^{T}\left(j_{i}^{z}-j_{i}^{(B) z}\right) .
\end{aligned}
$$

A current $j_{i}^{(B) \mu}=\left(n_{i}^{(B)}, j_{i}^{(B) z}\right)$ in the presence of a CP-violating Higgs background (or $\mathrm{CP}$-violating force $F^{\mu}$ ) but where no interactions with the plasma fields were considered was computed in Ref. [39]. It should satisfy the set of Boltzmann equations

$$
-v_{\omega}\left(n_{i}^{(B)}\right)^{\prime}+\left(j_{i}^{(B) z}\right)^{\prime}+\left\langle\left(F_{i}^{0}+v_{\omega} F_{i}^{z}\right) g\left(p_{z}, z\right) \frac{\partial f_{0}}{\partial E}\right\rangle=0,
$$




$$
D_{i} \Gamma_{i}^{T}\left(n_{i}^{(B)}\right)^{\prime}-v_{\omega}\left(j_{i}^{(B) z}\right)^{\prime}+\left\langle\frac{p_{z}}{E}\left(F_{i}^{0}+v_{\omega} F_{i}^{z}\right) g\left(p_{z}, z\right) \frac{\partial f_{0}}{\partial E}\right\rangle=0 .
$$

We can see from these equations, and in particular from (2.17) that the roles played by the CP-violating forces and currents $\left(n_{i}^{(B)}, j_{i}^{(B)}\right)$ are equivalent. In fact by subtracting (2.17) from (2.15) one obtains an equation

$$
-v_{\omega}\left(n_{i}-n_{i}^{(B)}\right)^{\prime}+\left(j_{i}^{z}-j_{i}^{(B) z}\right)^{\prime}+\Gamma_{i j} \frac{n_{j}}{k_{j}}=0,
$$

where the force terms have been replaced by the divergence of the $\mathrm{CP}$-violating current.

By subtracting now (2.18) from (2.16) we obtain

$$
\begin{aligned}
\left(j_{i}^{z}-j_{i}^{(B) z}\right) & =D_{i}\left[\left(n_{i}-n_{i}^{(B)}\right)^{\prime}-\frac{v_{\omega}}{\Gamma_{i}^{T}}\left(n_{i}-n_{i}^{(B)}\right)^{\prime \prime}+\mathcal{O}\left(v_{w}^{2}\right)\right] \\
& \simeq D_{i}\left(n_{i}-n_{i}^{(B)}\right)^{\prime},
\end{aligned}
$$

where the last approximation comes from the requirement $v_{\omega} / \Gamma_{i}^{T} L_{w} \ll 1$ (where $L_{w}$ is the wall thickness), which is necessary for the validity of the derivative expansion we will use in the calculation of the CP-violating currents in Section 3. Eq. (2.20) represents the well-known empirical law of diffusion or Fick's law.

Replacing now Eq. (2.20) into (2.19) we obtain the final expression for the diffusion equation as

$$
-v_{\omega} n_{i}^{\prime}+D_{i} n_{i}^{\prime \prime}+\Gamma_{i j} \frac{n_{j}}{k_{j}}=S_{i}\left[n^{(B)}\right],
$$

where the source is given by

$$
S_{i}=D_{i}\left(n_{i}^{(B)}\right)^{\prime \prime}-v_{\omega}\left(n_{i}^{(B)}\right)^{\prime} \simeq D_{i}\left(n_{i}^{(B)}\right)^{\prime \prime}
$$

and the last approximation comes from the fact that $D_{i} \gg L_{\omega} v_{\omega}$. Notice that Eq. (2.21) has the correct boundary conditions since in the absence of inelastic reactions (for $\Gamma_{i j}=0$ ) it provides the trivial solution $n_{i}=n_{i}^{(B)}$ as required.

We can compare this result with the source obtained in Ref. [41]. These authors work in a WKB approximation with quasi-particles. Within their semiclassical approximation the energy is a (constant) label of WKB-states and therefore the time component of the force $F_{i}^{0}=d E / d t$ vanishes, while they have assumed that $g\left(p_{z}, z\right)=1$. Moreover their source, $S_{i}^{C J K}$, is given by [41]

$$
S_{i}^{C J K}=-\frac{1}{\Gamma_{i}^{T}}\left\langle\frac{p_{z}}{E} v_{\omega} F_{i}^{z} \frac{\partial f_{0}}{\partial E}\right\rangle^{\prime}
$$

which appears in Eq. (2.18) above and can therefore be related to $n_{i}^{(B)}$ and $j_{i}^{(B) z}$ as they also appear in (2.18). Although we have obtained the sources $S_{i}$ in a way independent of the form of the CP-violating forces $F_{i}^{z}$ and $F_{i}^{0}$, for vanishing $F_{i}^{0}$ we obtain a relation between the sources $S_{i}$ and $S_{i}^{C J K}$. Indeed, using Eq. (2.18), we obtain

$$
S_{i}^{C J K} \simeq D_{i}\left(n_{i}^{(B)}\right)^{\prime \prime}-\frac{v_{\omega}}{\Gamma_{i}^{T}}\left(j_{i}^{(B) z}\right)^{\prime} \simeq S_{i}
$$


where the last approximation relies on the relation $D_{i} \Gamma_{i}^{T} \gg 1$. The latter relation holds for the case of charginos in the MSSM, analyzed in Section 3.

\section{The sources}

In this section we will make contact between the source in (2.22), and in particular the current $j^{(B) \mu}$, and the Green function and will apply the formalism to the sector of charginos in the MSSM.

First consider a chiral fermion (say a right-handed one) in the presence of the non-trivial background. Its Green function $S^{(B)}(x, y)$ can be considered as function of the center-ofmass coordinate $z=(x+y) / 2$ and the relative coordinate $r=x-y$,

$$
S^{(B)}(z+r / 2, z-r / 2) \text {. }
$$

Since we will make a gradient expansion with respect to the coordinate $z$ we perform a Fourier transformation with respect to the relative coordinate $r$ as

$$
S^{(B)}(z ; p)=\int d^{4} r e^{i p \cdot r} S^{(B)}(z+r / 2, z-r / 2)
$$

which is called the Wigner representation of the Green function $S^{(B)}$.

By making the general decomposition

$$
S^{(B)}=\sigma^{\mu} S_{\mu}^{(B)}(z ; p)
$$

one can define the corresponding background current $j^{(B) \mu}$ as

$$
j^{(B) \mu}=\int \frac{d^{4} p}{(2 \pi)^{4}} S^{(B) \mu}
$$

and making the inverse Wigner transformation (3.1) it can be cast as

$$
j^{(B) \mu}=\frac{1}{2} \lim _{r \rightarrow 0} \operatorname{Tr} \sigma^{\mu} S^{(B)}(z+r / 2, z-r / 2) .
$$

The current can be written in an alternative equivalent form, that makes connection with the discussion in the previous section,

$$
j^{(B) \mu}=-\int \frac{d^{4} p}{(2 \pi)^{4}} p^{\mu} \nabla^{v} S_{v}^{(B)}(z ; p)=\int \frac{d^{4} p}{(2 \pi)^{4}} S_{\mu}^{(B)} .
$$

Under small perturbations the relevant component of the integrand is approximately dominated by $\delta$ functions imposing the dispersion relation between $p_{0}$ and the energy of the particle. Indeed, for a free particle in the presence of a non-trivial chemical potential, the Green functions (A.5) and (A.7) lead to

$$
S_{\mu}=i \frac{p_{\mu}}{p^{2}-m^{2}+i \epsilon}+2 \pi p_{\mu} f\left(p_{0}, \mu\right) \delta\left(p^{2}-m^{2}\right),
$$

where $p^{2}=p^{\mu} p_{\mu}$ and, since the momentum integral of the first term vanishes, the charge density is obtained from the integration of the second term. Under these conditions, 
Eqs. (3.3) and (3.4) are consistent with the definitions of the particle density $n^{(B)}$ and current $\vec{j}^{(B)}$ in (2.7).

For fermions of opposite chirality (say left-handed ones) the previous expressions hold just changing $\sigma^{\mu} \rightarrow \bar{\sigma}^{\mu}$. On the other hand for several flavors, the mass eigenstates are an admixture of the weak eigenstates, with a mixing that depends on the value of the varying Higgs background. The expression of the diffusion equations and the sources, Eqs. (2.21) and (2.22), are related in a very simple way; namely the sources are trivially obtained by demanding a self-consistency relation in the absence of particle changing interactions. This property should hold, in first approximation, for any flavor structure of the theory. The relevant sources for the diffusion equations should be obtained by generalizing the trace in (3.4) to flavor space and eventually including a projection operator inside the trace, $\mathcal{P}$, when a given contribution is to be picked up. As stressed above, this formalism makes contact with the one proposed earlier by the authors of Ref. [41] and should lead to a more realistic estimate of the baryon asymmetry of the universe than the one presented in previous analyses of this subject.

Although, as we have already outlined, we agree with the results of Ref. [41] in the formal definition of the sources, we shall now show that the structure of these sources differs from the one presented by the authors of Ref. [41]. We shall try to clarify the origin of the discrepancy with those authors' results.

For the chargino sector of the MSSM we define the right-handed and left-handed fermions as

$$
\psi_{R}(x)=\left(\begin{array}{c}
\tilde{W}^{+} \\
\tilde{h}_{2}^{+}
\end{array}\right), \quad \psi_{L}(x)=\left(\begin{array}{c}
\tilde{W}^{-} \\
\tilde{h}_{1}^{-}
\end{array}\right)
$$

and expand the mass matrix to first order in derivatives around the point $z$ as

$$
M(x)=M(z)+(x-z)^{\mu} M_{\mu}(z),
$$

where we use the notation $M_{\mu}(z) \equiv \partial M(z) / \partial z^{\mu}$, and the mass matrix $M(z)$ is defined in (A.1).

We now consider the first term in (3.7) as part of the unperturbed Lagrangian, and the second term as the perturbation

$$
\mathcal{L}_{\text {int }}(x)=(x-z)^{\mu}\left\{\psi_{R}^{\dagger}(x) M_{\mu}(z) \psi_{L}(x)+\psi_{L}^{\dagger}(x) M_{\mu}^{\dagger}(z) \psi_{R}(x)\right\}
$$

or, in the basis of mass eigenstates,

$$
\begin{aligned}
\chi_{R}(x)= & \mathcal{U}(z) \psi_{R}(x), \quad \chi_{L}(x)=\mathcal{V}(z) \psi_{L}(x), \\
\mathcal{L}_{\text {int }}(x)= & (x-z)^{\mu}\left\{\chi_{R}^{\dagger}(x) \mathcal{U}(z) M_{\mu}(z) \mathcal{V}^{\dagger}(z) \chi_{L}(x)\right. \\
& \left.+\chi_{L}^{\dagger}(x) \mathcal{V}(z) M_{\mu}^{\dagger}(z) \mathcal{U}^{\dagger}(z) \chi_{R}(x)\right\} .
\end{aligned}
$$

We denote by $S^{L L}, S^{R R}, S^{L R}$ and $S^{R L}$ the left-left, right-right, left-right and right-left Green functions of free fermions in the mass eigenstate basis with mass $m_{i}(z)$ as given in (A.5). In this basis the right- and left-handed Green functions get modified to $S_{\chi}^{R R}$ and $S_{\chi}^{L L}$ by the presence of the interaction term (3.9) and can be defined as solutions of SchwingerDyson equations. To first order in the insertion given by the interaction term (3.9) the 
solution to the Schwinger-Dyson equations is

$$
\begin{aligned}
& S_{\chi}^{R R}(x, y ; z)=S^{R R}(x, y ; z)+\Delta S_{\chi}^{R R}(x, y ; z) \\
& \Delta S_{\chi}^{R R}(x, y ; z) \\
& \quad=\int d^{4} w(w-z)^{\mu} S^{R R}(x, w ; z) \mathcal{U}(z) M_{\mu}(z) \mathcal{V}^{\dagger}(z) S^{L R}(w, y ; z) \\
& \quad+\int d^{4} w(w-z)^{\mu} S^{R L}(x, w ; z) \mathcal{V}(z) M_{\mu}^{\dagger}(z) \mathcal{U}^{\dagger}(z) S^{R R}(w, y ; z)
\end{aligned}
$$

and a similar solution for $S_{\chi}^{L L}$.

The CP-violating Green function $\Delta S_{\chi}^{R R}(x, y ; z)$ defined in (3.10) can be written as

$$
\begin{aligned}
\Delta S_{\chi}^{R R}= & \frac{1}{4} \int \frac{d^{4} p}{(2 \pi)^{4}} e^{i p \cdot r}\left\{\sigma^{\mu} G \mathcal{U} M_{\mu} M^{\dagger} \mathcal{U}^{\dagger} G\right. \\
& \left.+\sigma_{\nu} p^{\nu} G^{\mu} \mathcal{U}\left(M M_{\mu}^{\dagger}+M_{\mu} M^{\dagger}\right) \mathcal{U}^{\dagger} G-\text { h.c. }\right\}
\end{aligned}
$$

where the notation $G^{\mu}=\partial G / \partial p_{\mu}$ has been used.

Working out Eq. (3.11) it can be cast as

$$
\begin{aligned}
\Delta S_{\chi}^{R R}= & \frac{1}{4} \int \frac{d^{4} p}{(2 \pi)^{4}} e^{i p \cdot r}\left\{\sigma_{\mu}\left(\begin{array}{cc}
G_{1}^{2} \mathcal{A}_{11}^{\mu} & G_{1} G_{2} \mathcal{A}_{12}^{\mu} \\
-G_{1} G_{2} \mathcal{A}_{12}^{\mu *} & -G_{2}^{2} \mathcal{A}_{11}^{\mu}
\end{array}\right)\right. \\
& \left.+(\sigma \cdot p)\left(G_{1 \mu} G_{2}-G_{1} G_{2 \mu}\right)\left(\begin{array}{cc}
0 & \mathcal{B}_{12}^{\mu} \\
-\mathcal{B}_{12}^{\mu *} & 0
\end{array}\right)\right\} .
\end{aligned}
$$

The calculation of the coefficients $\mathcal{A}_{11}^{\mu}, \mathcal{A}_{12}^{\mu}$ and $\mathcal{B}_{12}^{\mu}$ is straightforward. We will only give the relevant pieces that contribute to the $\mathrm{CP}$ violating current (3.3)

$$
\begin{aligned}
& \operatorname{Im}\left[\mathcal{A}_{11}^{\mu}\right]=-\frac{\operatorname{Im}\left(M_{2} \mu\right)}{\Lambda}\left(u_{1} u_{2}^{\mu}+u_{2} u_{1}^{\mu}\right), \\
& \operatorname{Im}\left[\left(M_{2} u_{1}+\mu u_{2}\right) \mathcal{A}_{12}^{\mu}\right]=-\Delta \frac{\operatorname{Im}\left(M_{2} \mu\right)}{\Lambda}\left(u_{1} u_{2}^{\mu}+u_{2} u_{1}^{\mu}\right), \\
& \operatorname{Im}\left[\left(M_{2} u_{1}+\mu u_{2}\right) \mathcal{B}_{12}^{\mu}\right]=-\operatorname{Im}\left(M_{2} \mu\right)\left(u_{1} u_{2}^{\mu}-u_{2} u_{1}^{\mu}\right) .
\end{aligned}
$$

We can see from (3.13) that the combination $u_{1} u_{2}^{\mu}-u_{2} u_{1}^{\mu}$ appears in the off-diagonal coefficients of $\Delta S_{\chi}^{R R}$. Had we neglected the latter we would have obtained only the orthogonal combination $u_{1} u_{2}^{\mu}+u_{2} u_{1}^{\mu}$ as claimed in Ref. [41]. However, contrary to the claim made by these authors, we do not find that the off-diagonal terms lead to a contribution of second-order to the currents relevant for electroweak baryogenesis. Since this is a crucial difference between our results and those obtained in Refs. [41,42], we will be more explicit about the origin of the discrepancy with those authors at the end of this section.

Returning to the derivation of the currents, the last step would be going from the Green function (3.12) to the CP-violating current as defined in (3.4). To this end we must rotate to the weak eigenstate basis and project onto the higgsino component. We must then define the Green function

$$
S_{R R}^{(B)}=\mathcal{U}^{\dagger} \Delta S_{\chi}^{R R} \mathcal{U}
$$


and the CP-violating background current (3.4) is now given by

$$
j_{R R}^{(B) \mu}=\lim _{r \rightarrow 0} \operatorname{Tr} \mathcal{P}_{22} \sigma^{\mu} S_{R R}^{(B)}(z+r / 2, z-r / 2),
$$

where $\mathcal{P}_{22}=\left(\sigma_{0}-\sigma_{3}\right) / 2$ with $\sigma_{i}$ being the two by two Pauli matrices in flavor space and $\sigma_{0}$ being the two by two identity matrix. The current $j_{R R}^{(B) \mu}$ can be given by the expression

$$
\begin{aligned}
j_{R R}^{(B) \mu}= & \frac{1}{4 \Lambda} \int \frac{d^{4} p}{(2 \pi)^{4}}\left\{\left[(\Lambda-\Delta) G_{1}^{2}-(\Lambda+\Delta) G_{2}^{2}\right] \operatorname{Im} \mathcal{A}_{11}^{\mu}\right. \\
& +\left(G_{1} G_{2}+G_{2} G_{1}\right) \operatorname{Im}\left[\left(M_{2} u_{1}+\mu u_{2}\right) \mathcal{A}_{12}^{\mu}\right] \\
& \left.+2 p^{\mu}\left(G_{1 v} G_{2}-G_{1} G_{2 v}\right) \operatorname{Im}\left[\left(M_{2} u_{1}+\mu u_{2}\right) \mathcal{B}_{12}^{v}\right]\right\},
\end{aligned}
$$

where the coefficients $\mathcal{A}_{11}, \mathcal{A}_{12}$ and $\mathcal{B}_{12}$ of the Green function in (3.13) are made explicit. The final expression can be written as

$$
\begin{aligned}
j_{R R}^{(B) \mu}= & -\frac{\operatorname{Im}\left(M_{2} \mu\right)}{4 \Lambda} \int \frac{d^{4} p}{(2 \pi)^{4}}\left\{\left[G_{1}^{2}-G_{2}^{2}-\frac{\Delta}{\Lambda}\left(G_{1}-G_{2}\right)^{2}\right]\left(u_{1} u_{2}^{\mu}+u_{2} u_{1}^{\mu}\right)\right. \\
& \left.+2 p^{\mu}\left(G_{1 v} G_{2}-G_{1} G_{2 v}\right)\left(u_{1} u_{2}^{v}-u_{2} u_{1}^{\nu}\right)\right\} .
\end{aligned}
$$

The left-handed current $j_{L L}^{(B) \mu}$ can be obtained in the same way. The corresponding result can be read off from (3.17) after making the changes $\Delta \rightarrow \bar{\Delta}$ and $u_{1} \leftrightarrow u_{2}$. From them the vector and axial currents can be computed and the result obtained from (3.17) agrees with that presented in Eqs. (3.14) and (3.15) of Ref. [39].

To conclude this section we would like to make some final comments concerning the origin of our discrepancy with the results presented in Ref. [42]. In Ref. [42] it is correctly argued that, in the absence of particle-changing interactions and in the mass eigenstate basis at a given point $z$, terms proportional to $u_{1} u_{2}^{\mu}-u_{2} u_{1}^{\mu}$ only appear in the off-diagonal elements of the two-by-two matrix of Green functions. This is in agreement with the result obtained in Eq. (3.12). From here, the authors of Ref. [42] conclude that the sources receive no contribution proportional to $u_{1} u_{2}^{\mu}-u_{2} u_{1}^{\mu}$ at first order in the derivative expansion. This conclusion is not correct as we will now explain.

First of all, let us emphasize that the particle changing interactions are essential in order to convert the original higgsino density into a left-handed quark one. In the absence of these interactions with the plasma the net result for the baryon asymmetry will be much smaller than the one required for consistency with big-bang nucleosynthesis. The higgsino states cannot be associated with the mass eigenstates at any point $z$ along the bubble wall, where the Higgs background is non-vanishing. In order to treat the particle changing interactions one has to transform to the weak interaction basis. Of course it would be very exciting "in a full quantum formulation of the diffusion equations" if the collision terms would project onto quasi-classical flavor states. A proof of this statement (which is outside the scope of the present paper) would follow similar lines to the ones in Ref. [42].

Second, observe that the currents are completely determined by $\Delta S_{\chi}^{R R}\left(\Delta S_{\chi}^{L L}\right)$. The dominant, diagonal contribution $S^{R R}\left(S^{L L}\right)$ plays no role in the definition of the CPviolating densities at this order. The $\mathrm{CP}$-violating densities receive contributions from 
both the diagonal and off-diagonal terms after rotating to the weak eigenstate basis as in (3.14) and both contributions appear at first order. Therefore, one should compute both the diagonal and off-diagonal contribution to the Green functions. The fact that the diagonal terms have no dependence on the combination $u_{2} u_{1}^{\mu}-u_{1} u_{2}^{\mu}$ does not imply that the sources share such an independence, since the off-diagonal terms in the mass eigenstate basis play an important role when interactions are included. Actually, as shown in Ref. [39], depending on the parameter space, the contributions proportional to $u_{1} u_{2}^{\mu}-u_{2} u_{1}^{\mu}$ may be the dominant ones in the generation of a relevant baryon asymmetry of the universe.

Similar arguments can be used to show that the considerations of the authors of Ref. [41], leading to the absence of a dependence of the sources on $u_{2} u_{1}^{\mu}-u_{1} u_{2}^{\mu}$ cannot be sustained. Indeed, Ref. [42] tries to provide a formal proof to these considerations. Moreover, the authors of Ref. [42] neglect the chargino mixing in the interaction terms, in spite of the fact that mixing is essential for a non-vanishing source. Therefore, their treatment relies on an approximation that is invalid in the interesting region where $M_{2}$ is close to $\mu$. As we showed in Ref. [39] it is precisely in this region of parameters where the sources proportional to $u_{2} u_{1}^{\mu}-u_{1} u_{2}^{\mu}$ become most relevant in the baryon asymmetry generation (see discussion below).

\section{Implications for the baryon asymmetry}

The detailed evaluation of the currents necessary to compute the sources for the diffusion equations presented in the last section was already provided in a previous article [39]. In Ref. [39], the resulting baryon asymmetry was computed under the assumption that the connection between the sources for the diffusion equations and the CPviolating currents was that presented in Refs. [29,33], namely $S_{i}=\Gamma_{i}^{T} n_{i}^{(B)}$. In Section 2 we showed that, contrary to this assumption, the real sources, Eq. (2.22), have a form that depends on derivatives of the CP-violating currents computed in the presence of the background fields. Therefore, the resulting value of the baryon asymmetry is different from the one computed in Ref. [39].

The baryon asymmetry resulting from the solution of the diffusion equations depends on integral functions along the wall of the temperature dependent value of the sources times exponential factors which are slowly varying along the bubble wall [39]. Therefore, the new values can be simply obtained from the earlier ones by integration by parts, taking care of the proper boundary conditions.

As discussed in the introduction, the total baryon asymmetry depends on two different contributions. The first one is proportional to

$$
\epsilon_{i j} H_{i} \partial_{\mu} H_{j}=v^{2}(T) \partial_{\mu}(\beta) .
$$

This expression, Eq. (4.1), is proportional to the variation of the angle

$$
\beta=\arctan \left[v_{2}(T) / v_{1}(T)\right]
$$

at the wall of the expanding bubble, which tends to zero for large values of $m_{A}$. Furthermore, independently of $m_{A}$, for large values of $\tan \beta, \beta$ varies only slightly from 
its value $\beta \simeq \pi / 2$ and therefore the baryon asymmetry tends to be suppressed. Finally, this contribution to the sources of the baryon asymmetry has a resonant behaviour for $M_{2}=|\mu|$. Therefore, it becomes more relevant for moderate values of $m_{A}$ and $\tan \beta$ and for values of $M_{2} \simeq|\mu|$.

The second contribution to the baryon asymmetry depends on

$$
H_{1} \partial_{\mu} H_{2}+H_{2} \partial_{\mu} H_{1}=v^{2} \cos (2 \beta) \partial_{\mu} \beta+v \partial_{\mu} v \sin (2 \beta) .
$$

Similarly to (4.1), the first term in the expression (4.2) is suppressed for large values of $m_{A}$ and/or $\tan \beta$. Although the second term is also suppressed for large values of $\tan \beta$, it is unsuppressed for large values of $m_{A}$. Therefore, we expect this contribution to become the dominant one for large values of $m_{A}$, particularly in the non-resonant regions with values of $M_{2}$ very different from $|\mu|$.

Finally, let us remark that the final baryon asymmetry is obtained by solving the diffusion equation for the total baryon number. This equation may be easily derived by summing up the diffusion equations for the thirty six chiral quarks of the Standard Model (three generations, two flavors of quark per generation, two chiralities and three colors per quark). The sum of the quark densities is just three times the total baryon number density (each quark carries a baryon number 1/3). The variation of the baryon number is governed by the sphaleron processes, which affect only the left-handed chiral quarks and leptons.

The left-handed quark chemical potentials receive two contributions. The dominant one may be obtained by solving the diffusion equation for the different colors of quarks in the presence of gauge, Yukawa, mass and strong sphaleron interactions. Since no baryon number violating processes are included, the solutions to these diffusion equations lead to an equal number of baryons of a given chirality and antibaryons of the opposite chirality. These dominant densities may be considered as approximately constant during the characteristic long times in which the weak sphaleron processes take place.

The left-handed densities receive also a subdominant contribution coming from the weak sphaleron interactions. This contribution is associated with a net-baryon number which, considering effective mixing between the different flavors and colors of quarks, is shared in approximately equal parts by all 36 of them. Finally, there is also a net lepton number created which due to charge conservation is equal to the net baryon number.

Taking all these considerations into account, and considering the right-handed leptons out of equilibrium, one arrives to the equation

$$
D n_{B}^{\prime \prime}(z)-v_{\omega} n_{B}^{\prime}(z)=\theta(-z) \Gamma_{w s}\left(\frac{3 T^{2} \mu_{L}^{\mathrm{diff}}(z)}{4}+A n_{B}(z)\right),
$$

where $\Gamma_{w s}=6 k_{w s} \alpha_{w}^{5} T$ ( $\alpha_{w}$ is the weak coupling constant), with $k_{w s} \simeq 20$ [4] being the weak sphaleron rate, while $\mu_{L}^{\text {diff }}$ is equal to the sum over the three generations of the lefthanded up and down quark chemical potentials associated with a given color of quarks, as obtained by solving their diffusion equations in the absence of the slow sphaleron interactions. In the numerical simulations, we have considered heavy all squarks and sleptons except for the lightest stop, in which case the coefficient $A=24 / 7$, which differs only slightly from the SM result $A^{\mathrm{SM}}=15 / 4$ (see also Ref. [41]).

In Fig. 1 we show the comparison of the baryon number to entropy ratio $\eta$ computed within the present model with the mean value of the one consistent with big-bang 


$$
\mathrm{M}_{2}=\mu
$$

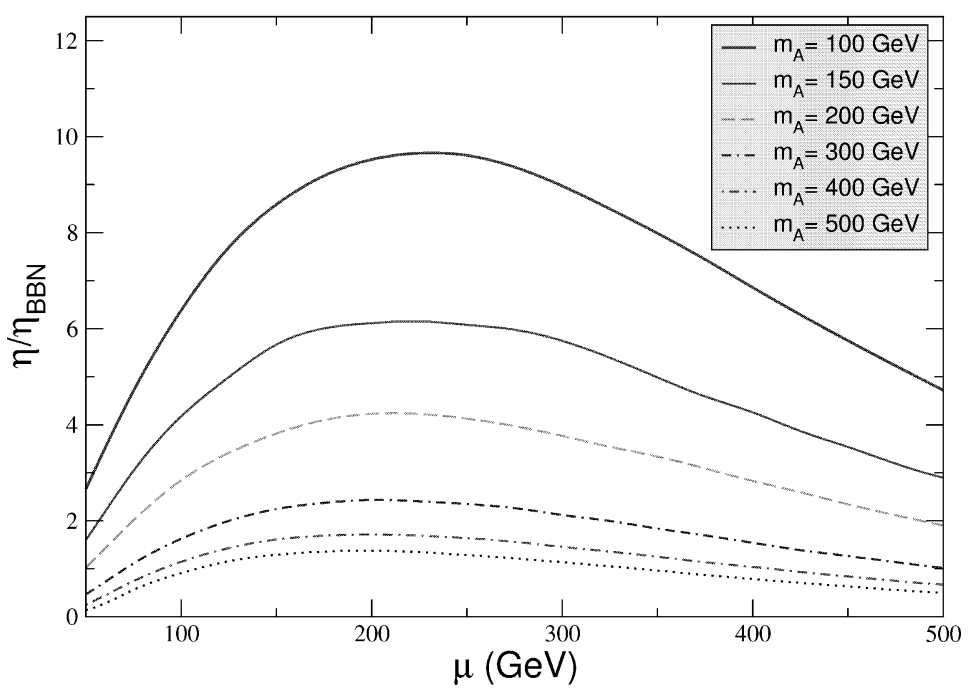

Fig. 1. Plot of $\eta / \eta_{\mathrm{BBN}}$ as a function of $\mu$ for $\tan \beta=10, M_{2}=\mu$ and the specified values of $m_{A}$.

nucleosynthesis $(\mathrm{BBN}), \eta_{\mathrm{BBN}} \simeq(6 \pm 3) \times 10^{-11}$ [43]. We have chosen soft supersymmetry breaking parameters in the stop sector such that they lead to a value of the Higgs boson mass consistent with the present experimental constraints, and the lightest stop light enough so that the phase transition becomes strongly first order, $v(T) / T \gtrsim 1$ [44].

Since the dominant component of the sources comes from the chargino sector [30,39], the results of the baryon asymmetry depend only indirectly on the stop parameters, through the values of $v(T) / T$ and $\beta(T)$. Different choices of the parameters lead to variations of the final result by a factor of order one. Since the method of computation has implicit uncertainties of similar order, so far $v(T) / T \gtrsim 1$, the specific choice of the parameters does not affect the results for the baryon asymmetry in any significant way.

We have chosen a maximal value of the phase of the $\mu$ parameter, $\sin \phi_{\mu}=1$. Therefore, the inverse of the values shown in the figure can be interpreted as the value of $\sin \phi_{\mu}$ necessary to obtain a prediction consistent with BBN. Observe that due to the uncertainty in the value of $\eta_{\mathrm{BBN}}$ [43], the value of $\eta_{\mathrm{BBN}}$ may be a factor 2 smaller than the above quoted mean value and therefore the value of the phase may be a factor 2 smaller than the value obtained by the procedure described above. ${ }^{2}$ Hence, from Fig. 1 we see that for $m_{A}$ consistent with the present experimental constraints, values of $\sin \phi_{\mu} \gtrsim 0.05$ are preferred.

In Fig. 2 we present the values of the ratio $\eta / \eta_{\mathrm{BBN}}$ as a function of $\mu$, for a fixed value of $M_{2}=200 \mathrm{GeV}$. For small values of $m_{A}$ the dominant contribution comes from the resonant behavior at $M_{2}=|\mu|$. However, as $m_{A}$ increases the maximum of $|\eta|$ is obtained for values

\footnotetext{
${ }^{2}$ An additional reduction factor on $\sin \phi_{\mu}$ could come from the contribution of neutralinos to $\eta$, that has not been considered in this paper.
} 


$$
M_{2}=200 \mathrm{GeV}
$$

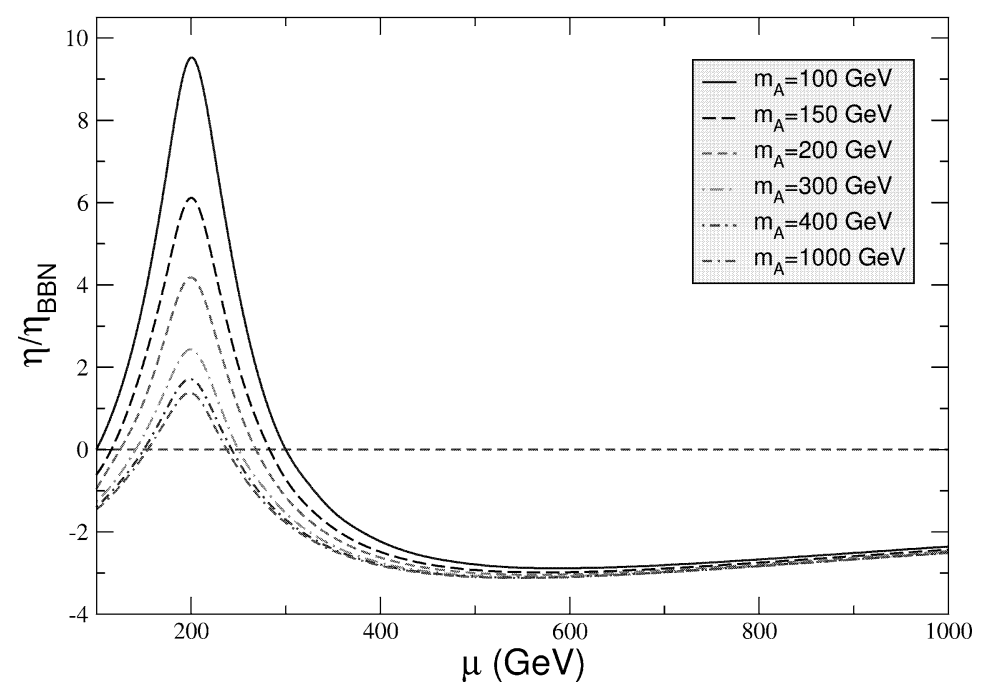

Fig. 2. Plot of $\eta / \eta_{\mathrm{BBN}}$ as a function of $\mu$ for $M_{2}=200 \mathrm{GeV}, \tan \beta=10$, and the specified values of $m_{A}$.

of $\mu$ much larger than $M_{2}$. The dominant, non-resonant component has opposite sign to the resonant one and for large values of $\mu$ compared to $M_{2}$, it becomes only slightly dependent on $m_{A}$, in agreement with our discussion above. We see that even for large values of $m_{A}$, values of the baryon asymmetry consistent with BBN predictions may be obtained for phases of order one. Observe that the baryon asymmetry tends to zero for large values of $\mu$, since the higgsino component of the lightest chargino, present in the plasma, tends to zero in this limit.

The above results are important in view of the constraints coming from electric dipole moments [46]. It has been recently observed that, even in the presence of very heavy squarks and leptons, the electric dipole moment contributions may not be small, due to the existence of two loop diagrams involving chargino loops and the non-SM like Higgs bosons [47]. These contributions are enhanced with increasing $\tan \beta$, but become rapidly small for large values of $m_{A}$ and small mixing in the chargino sector. The results of Fig. 2 show that a relevant baryon asymmetry may be obtained even in this particular regime of parameters.

\section{Conclusions}

In this article, we have derived the diffusion equations governing the result for the baryon density generated by the passage of the expanding wall at temperatures close to the electroweak phase transition temperature. Important for this result was the identification of the sources for the diffusion equations in terms of the currents generated by the non-trivial Higgs backgrounds, which vary along the bubble wall. We demonstrated that there are 
two different contributions to these sources, one proportional to $\epsilon^{i j} H_{i} \partial_{\mu} H_{j}$ and the other proportional to $H_{1} \partial_{\mu} H_{2}+(2 \rightarrow 1)$. Contrary to previous claims, we showed that none of these contributions to the sources vanish. We clarified the origin of the discrepancy of our results with those of Refs. [41,42].

The two contributions to the sources have a different dependence on the parameters of the minimal supersymmetric standard model. While the former contribution has a resonant behaviour for $M_{2}=|\mu|$ and goes to zero for large values of $m_{A}$, the latter has no resonant behaviour and remains non-vanishing for large values of $m_{A}$.

The final result for the baryon asymmetry shows that consistency with Big Bang Nucleosynthesis demands the relative phase between $\mu$ and the gaugino mass parameter $M_{2}$ (or $M_{1}$, if we consider the neutralino contribution) to be sizeable. Our calculations lead to a bound on the value of the CP-violating phase, $\sin \phi \gtrsim 0.05$, but due to the natural uncertainties associated with our simplified treatment of the baryon asymmetry calculation, and the experimental error in the determination of $\eta_{\mathrm{BBN}}$, we cannot reliably rule out the possibility that somewhat smaller values of the phase $\phi_{\mu}$ may lead to consistency with the BBN predictions.

On the other hand, for sizeable values of the CP-violating phase $\sin \phi_{\mu}$, cancellation of the potentially large electric dipole moment contributions is required. These contributions are dominated by one-loop effects with scalar particles and gauginos participating in the loop. These corrections may be efficiently suppressed for large values of the scalar masses of the first and second generation [46]. Under these conditions, non-vanishing corrections still appear at the two-loop level [47]. The most relevant two-loop corrections are enhanced for large values of $\tan \beta$, but are suppressed for large values of the CP-odd mass parameter $m_{A}$. It is reassuring to observe that even for large values of the CP-odd mass parameter a baryon asymmetry consistent with BBN may be obtained.

In summary within the framework of the minimal supersymmetric extension of the Standard Model, the realization of the electroweak baryogenesis scenario requires the presence of a light Higgs boson, with mass smaller than about $120 \mathrm{GeV}$, and a light stop, with mass smaller than the top quark mass [11-28,44]. Moreover it has been found that this baryogenesis mechanism does not guarantee a new $\mathrm{CP}$ violating signal at the $B$ factories [48]. Therefore, apart from the experimental constraints coming from electric dipole moments, a definitive test of the electroweak scenario within the MSSM will come from Higgs [49] and stop [50] searches at the Tevatron and at the LHC.

\section{Acknowledgements}

We acknowledge discussions with T. Prokopec and S. Weinstock. M.Q. and M.S. would like to thank the Theory Division of the Fermi National Accelerator Laboratory, where part of this work was done, for hospitality. M.C. and C.W. also wish to thank the CERN Theory Division, where part of this work was done, for hospitality. M.S. would like to thank the Theory Group of Instituto de Estructura de la Materia, where this work was partly done, for hospitality. M.Q. would also like to thank the Physics and Astronomy Department of the Johns Hopkins University for the hospitality extended to him as Bearden Visiting Professor. The work of C.W. is supported in part by the US DOE, Div. of HEP, Contract 
W-31-109-ENG-38. Fermilab is operated by Universities Research Association Inc. under Contract No. DE-AC02-76CH02000 with the DOE. The work of M.S. is supported by the US DOE Contract DE-A505-89ER40518.

\section{Appendix A. The chargino sector}

In this appendix we summarize some useful formulae which concern the chargino sector of the MSSM. The chargino mass matrix is given by

$$
M(z)=\left(\begin{array}{cc}
M_{2} & u_{2}(z) \\
u_{1}(z) & \mu_{c}
\end{array}\right)
$$

where we have defined $u_{i}(z) \equiv g H_{i}(z)$. The diagonalizing matrices are

$$
\begin{aligned}
& \mathcal{U}=\frac{1}{\sqrt{2 \Lambda(\Delta+\Lambda)}}\left(\begin{array}{cc}
\Delta+\Lambda & M_{2} u_{1}+\mu_{c}^{*} u_{2} \\
-\left(M_{2} u_{1}+\mu_{c} u_{2}\right) & \Delta+\Lambda
\end{array}\right), \\
& \mathcal{V}=\frac{1}{\sqrt{2 \Lambda(\bar{\Delta}+\Lambda)}}\left(\begin{array}{cc}
\Delta+\Lambda & M_{2} u_{2}+\mu_{c} u_{1} \\
-\left(M_{2} u_{2}+\mu_{c}^{*} u_{1}\right) & \bar{\Delta}+\Lambda
\end{array}\right),
\end{aligned}
$$

where field redefinitions have been made in order to make the Higgs vacuum expectation values, as well as the weak gaugino mass $M_{2}$, real,

$$
\begin{aligned}
& \Delta=\left(M_{2}^{2}-\left|\mu_{c}\right|^{2}-u_{1}^{2}+u_{2}^{2}\right) / 2, \\
& \bar{\Delta}=\left(M_{2}^{2}-\left|\mu_{c}\right|^{2}-u_{2}^{2}+u_{1}^{2}\right) / 2, \\
& \Lambda=\left(\Delta^{2}+\left|M_{2} u_{1}+\mu_{c}^{*} u_{2}\right|^{2}\right)^{1 / 2},
\end{aligned}
$$

and the mass eigenvalues are given by

$$
\begin{aligned}
& m_{1}(z)=\frac{\left(\Delta+\Lambda+u_{1}^{2}(z)\right) M_{2}+u_{1}(z) u_{2}(z) \mu_{c}^{*}}{\sqrt{(\Delta+\Lambda)(\bar{\Delta}+\Lambda)}}, \\
& m_{2}(z)=\frac{\left(\Delta+\Lambda-u_{2}^{2}(z)\right) \mu_{c}-u_{1}(z) u_{2}(z) M_{2}}{\sqrt{(\Delta+\Lambda)(\bar{\Delta}+\Lambda)}} .
\end{aligned}
$$

In the mass eigenstate basis the free fermionic Green functions can be written in terms of the bosonic ones $G(p ; z)$ as

$$
\begin{aligned}
& S^{R R}(p ; z)=\sigma_{\mu} p^{\mu} G(p ; z), \\
& S^{R L}(p ; z)=\left(\begin{array}{cc}
m_{1}(z) & 0 \\
0 & m_{2}(z)
\end{array}\right) G(p ; z), \\
& S^{L R}(p ; z)=\left(\begin{array}{cc}
m_{1}^{*}(z) & 0 \\
0 & m_{2}^{*}(z)
\end{array}\right) G(p ; z), \\
& S^{L L}(p ; z)=\bar{\sigma}_{\mu} p^{\mu} G(p ; z)
\end{aligned}
$$

and

$$
G=\left(\begin{array}{cc}
G_{1}(p ; z) & 0 \\
0 & G_{2}(p ; z)
\end{array}\right)
$$


where $G_{i}$ is the two-by-two matrix of a bosonic propagator corresponding to the mass $\left|m_{i}(z)\right|$ (A.4) given by

$$
\begin{aligned}
G_{i}^{11} & =P_{i}^{+}-f_{F}\left(P_{i}^{+}-P_{i}^{-}\right), \\
G_{i}^{12} & =\left[\theta\left(p^{0}\right)-f_{F}\right]\left(P_{i}^{+}-P_{i}^{-}\right), \\
G_{i}^{21} & =\left[\theta\left(-p^{0}\right)-f_{F}\right]\left(P_{i}^{+}-P_{i}^{-}\right), \\
G_{i}^{22} & =-P_{i}^{-}-f_{F}\left(P_{i}^{+}-P_{i}^{-}\right),
\end{aligned}
$$

where $f_{F} \equiv n_{F}\left(\left|p^{0}\right|\right)$ is the Fermi-Dirac distribution function in equilibrium, which contains the dependence on the temperature $T$,

$$
P_{i}^{ \pm}=\frac{1}{p_{0}^{2}-\vec{p}^{2}-\left|m_{i}(z)\right|^{2} \pm 2 i \Gamma_{i}\left|p^{0}\right|},
$$

and $\Gamma_{i}$ is the particle width.

\section{References}

[1] For reviews, see A.G. Cohen, D.B. Kaplan, A.E. Nelson, Annu. Rev. Nucl. Part. Sci. 43 (1993) 27; M. Quirós, Helv. Phys. Acta 67 (1994) 451;

V.A. Rubakov, M.E. Shaposhnikov, Phys. Usp. 39 (1996) 461;

M. Carena, C.E.M. Wagner, hep-ph/9704347;

A. Riotto, M. Trodden, Annu. Rev. Nucl. Part. Sci. 49 (1999) 35;

M. Quirós, M. Seco, Nucl. Phys. B (Proc. Suppl.) 81 (2000) 63, hep-ph/9703274.

[2] A.D. Sakharov, JETPL 91B (1967) 24.

[3] G. 't Hooft, Phys. Rev. Lett. 37 (1976) 8;

G. 't Hooft, Phys. Rev. D 14 (1976) 3432.

[4] D. Bodeker, Phys. Lett. B 426 (1998) 351, hep-ph/9801430;

P. Arnold, L.G. Yaffe, hep-ph/9912306;

P. Arnold, Phys. Rev. D 62 (2000) 036003;

G.D. Moore, K. Rummukainen, Phys. Rev. D 61 (2000) 105008;

G.D. Moore, Phys. Rev. D 62 (2000) 085011.

[5] N.S. Manton, Phys. Rev. D 28 (1983) 2019;

F.R. Klinkhamer, N.S. Manton, Phys. Rev. D 30 (1984) 2212.

[6] A.G. Cohen, D.B. Kaplan, A.E. Nelson, Phys. Lett. B 336 (1994) 41, hep-ph/9406345.

[7] G.R. Farrar, M.E. Shaposhnikov, Phys. Rev. Lett. 70 (1993) 2833;

G.R. Farrar, M.E. Shaposhnikov, Phys. Rev. Lett. 71 (1993) 210, Erratum;

G.R. Farrar, M.E. Shaposhnikov, Phys. Rev. D 50 (1994) 774.

[8] M.B. Gavela, P. Hernández, J. Orloff, O. Pène, C. Quimbay, Nucl. Phys. B 430 (1994) 382;

P. Huet, E. Sather, Phys. Rev. D 51 (1995) 379.

[9] K. Jansen, Nucl. Phys. B (Proc. Suppl.) 47 (1996) 196, hep-lat/9509018;

K. Rummukainen, M. Tsypin, K. Kajantie, M. Laine, M. Shaposhnikov, Nucl. Phys. B 532 (1998) 283;

K. Rummukainen, K. Kajantie, M. Laine, M. Shaposhnikov, M. Tsypin, hep-ph/9809435.

[10] G.F. Giudice, Phys. Rev. D 45 (1992) 3177;

S. Myint, Phys. Lett. B 287 (1992) 325.

[11] J.R. Espinosa, M. Quirós, F. Zwirner, Phys. Lett. B 307 (1993) 106.

[12] A. Brignole, J.R. Espinosa, M. Quirós, F. Zwirner, Phys. Lett. B 324 (1994) 181.

[13] M. Carena, M. Quirós, C.E.M. Wagner, Phys. Lett. B 380 (1996) 81.

[14] D. Delepine, J.M. Gérard, R. González Felipe, J. Weyers, Phys. Lett. B 386 (1996) 183. 
[15] J.M. Moreno, D.H. Oaknin, M. Quirós, Nucl. Phys. B 483 (1997) 267;

J.M. Moreno, D.H. Oaknin, M. Quirós, Phys. Lett. B 395 (1997) 234.

[16] J. Cline, K. Kainulainen, Nucl. Phys. B 482 (1996) 73;

J. Cline, K. Kainulainen, Nucl. Phys. B 510 (1998) 88.

[17] M. Laine, Nucl. Phys. B 481 (1996) 43;

M. Losada, Phys. Rev. D 56 (1997) 2893;

G. Farrar, M. Losada, Phys. Lett. B 406 (1997) 60.

[18] J.R. Espinosa, Nucl. Phys. B 475 (1996) 273.

[19] B. de Carlos, J.R. Espinosa, Nucl. Phys. B 503 (1997) 24.

[20] D. Bodeker, P. John, M. Laine, M.G. Schmidt, Nucl. Phys. B 497 (1997) 387.

[21] M. Carena, M. Quirós, C.E.M. Wagner, Nucl. Phys. B 524 (1998) 3.

[22] M. Laine, K. Rummukainen, Nucl. Phys. B 535 (1998) 423.

[23] J.M. Moreno, M. Quirós, M. Seco, Nucl. Phys. B 526 (1998) 489.

[24] J.M. Cline, G.D. Moore, Phys. Rev. Lett. 81 (1998) 3315.

[25] P. John, Phys. Lett. B 452 (1999) 221;

S.J. Huber, P. John, M. Laine, M.G. Schmidt, Phys. Lett. B 475 (2000) 104.

[26] M. Losada, Nucl. Phys. B 537 (1999) 3;

M. Losada, Nucl. Phys. B 569 (2000) 125;

M. Laine, M. Losada, Nucl. Phys. B 582 (2000) 277.

[27] F. Csikor, Z. Fodor, P. Hegedus, A. Jakovac, S.D. Katz, A. Piroth, Phys. Rev. Lett. 85 (2000) 932.

[28] M. Laine, K. Rummukainen, Nucl. Phys. B 597 (2001) 23, hep-lat/0009025.

[29] P. Huet, A.E. Nelson, Phys. Lett. B 355 (1995) 229;

P. Huet, A.E. Nelson, Phys. Rev. D 53 (1996) 4578.

[30] M. Carena, M. Quirós, A. Riotto, I. Vilja, C.E.M. Wagner, Nucl. Phys. B 503 (1997) 387.

[31] J. Cline, M. Joyce, K. Kainulainen, Phys. Lett. B 417 (1998) 79.

[32] T. Multamaki, I. Vilja, Phys. Lett. B 411 (1997) 301.

[33] A. Riotto, Int. J. Mod. Phys. D 7 (1998) 815;

A. Riotto, Nucl. Phys. B 518 (1998) 339;

A. Riotto, Phys. Rev. D 58 (1998) 095009.

[34] M.P. Worah, Phys. Rev. D 56 (1997) 2010;

M.P. Worah, Phys. Rev. Lett. 79 (1997) 3810.

[35] H. Davoudiasl, K. Rajagopal, E. Westphal, Nucl. Phys. B 515 (1998) 384.

[36] K. Enqvist, A. Riotto, I. Vilja, Phys. Lett. B 438 (1998) 273.

[37] M. Trodden, Rev. Mod. Phys. 71 (1999) 1463.

[38] N. Rius, V. Sanz, Nucl. Phys. B 570 (2000) 155.

[39] M. Carena, J.M. Moreno, M. Quiros, M. Seco, C.E. Wagner, Nucl. Phys. B 599 (2001) 158.

[40] M. Joyce, T. Prokopec, N. Turok, Phys. Rev. Lett. 75 (1995) 1695;

M. Joyce, T. Prokopec, N. Turok, Phys. Rev. Lett. 75 (1995) 3375, hep-ph/9408339, Erratum;

M. Joyce, T. Prokopec, N. Turok, Phys. Rev. D 53 (1996) 2958, hep-ph/9410282.

[41] J.M. Cline, K. Kainulainen, Phys. Rev. Lett. 85 (2000) 5519;

J.M. Cline, M. Joyce, K. Kainulainen, JHEP 07 (2000) 018, hep-ph/0110031.

[42] K. Kainulainen, T. Prokopec, M.G. Schmidt, S. Weinstock, JHEP 0106 (2001) 031, hep-ph/0105295; K. Kainulainen, T. Prokopec, M.G. Schmidt, S. Weinstock, hep-ph/0202177.

[43] K. Hagiwara, et al., Particle Data Group, Phys. Rev. D 66 (2002) 010001.

[44] M. Quiros, Nucl. Phys. B (Proc. Suppl.) 101 (2001) 401, hep-ph/0101230.

[45] See, for example, S.R. De Groot, W.A. Van Leeuwen, C.G. Van Weert, Relativistic Kinetic Theory. Principles and Applications, North-Holland, Amsterdam, 1980.

[46] T. Ibrahim, P. Nath, Phys. Lett. B 418 (1998) 98;

T. Ibrahim, P. Nath, Phys. Rev. D 57 (1998) 478;

T. Ibrahim, P. Nath, Phys. Rev. D 58 (1998) 019901, Erratum;

T. Ibrahim, P. Nath, Phys. Rev. D 58 (1998) 111301;

T. Ibrahim, P. Nath, Phys. Rev. D 60 (1999) 099902, Erratum;

M. Brhlik, G.J. Good, G.L. Kane, Phys. Rev. D 59 (1999) 115004;

A. Pomarol, D. Tommasini, Nucl. Phys. B 466 (1996) 3; 
A.G. Cohen, D.B. Kaplan, A.E. Nelson, Phys. Lett. B 388 (1996) 588;

J. Feng, K. Matchev, F. Wilczek, Phys. Lett. B 482 (2000) 388;

D. Chang, W.-Y. Keung, A. Pilaftsis, Phys. Rev. Lett. 82 (1999) 900;

A. Pilaftsis, Phys. Lett. B 471 (1999) 174;

D. Chang, W.-F. Chang, W.-Y. Keung, Phys. Lett. B 478 (2000) 239;

A. Pilaftsis, Phys. Rev. D 62 (2000) 016007.

[47] D. Chang, W.F. Chang, W.Y. Keung, hep-ph/0205084;

A. Pilaftsis, hep-ph/0207277.

[48] H. Murayama, A. Pierce, hep-ph/0201261.

[49] M. Carena, et al., hep-ph/0010338;

D. Cavalli, et al., hep-ph/0203056;

M. Carena, H.E. Haber, Review on Higgs boson theory and phenomenology, preprint FERMILAB-Pub02/114-T, SCIPP 02/07.

[50] H. Baer, J. Sender, X. Tata, Phys. Rev. D 50 (1994) 4517;

R. Demina, J.D. Lykken, K.T. Matchev, A. Nomerotski, Phys. Rev. D 62 (2000) 035011;

C.L. Chou, M.E. Peskin, Phys. Rev. D 61 (2000) 055004;

S. Abel, et al., SUGRA Working Group Collaboration, hep-ph/0003154;

A. Djouadi, M. Guchait, Y. Mambrini, Phys. Rev. D 64 (2001) 095014;

M. Carena, D. Choudhury, R.A. Diaz, H.E. Logan, C.E. Wagner, hep-ph/0206167. 